\title{
Influence of Catalyst Pore Network Structure on the Hysteresis of Multiphase Reactions
}

\author{
Guanghua Ye a , Xinggui Zhou ${ }^{\mathrm{a},{ }^{*}}$, Marc-Olivier Coppens ${ }^{\mathrm{b}, \dagger}$, Jinghong Zhou ${ }^{\mathrm{a}}$, Weikang Yuan ${ }^{\mathrm{a}}$ \\ ${ }^{a}$ State Key Laboratory of Chemical Engineering, East China University of Science and \\ Technology, Shanghai 200237, China \\ ${ }^{\mathrm{b}}$ Department of Chemical Engineering, University College London, London WC1E 7JE, UK
}

\begin{abstract}
The effects of the catalyst pore network structure on multiphase reactions in catalyst pellets are investigated by using the experimentally validated pore network model proposed in our recent work (AIChE J. 2016, 62, 451). The simulations display hysteresis loops of the effectiveness factor. The hysteresis loop area becomes significantly larger, when having small volume-averaged pore radius, wide pore size distribution, and low pore connectivity; however, the loop area is insensitive to pellet size, even though it affects the value of the effectiveness factor. The hysteresis loop area is also strongly affected by the spatial distribution of the pore size, in particular for a bimodal pore size distribution. The pore network structure directly influences mass transfer, capillary condensation, and pore blocking, and subsequently passes these influences on to the hysteresis loop of the effectiveness factor. Recognizing these effects is essential when designing porous catalysts for multiphase reaction processes.
\end{abstract}

\footnotetext{
*First corresponding author. Tel.: +86-21-64253509. Fax.: +86-21-64253528. Email address: xgzhou@ecust.edu.cn

† Second corresponding author. Tel.: +44-20-31081126. Fax.: +44-20-73832348. Email address: m.coppens@ucl.ac.uk
} 
Keywords: Pore network structure, multiphase reaction, hysteresis, pore network model, capillary condensation

\section{Introduction}

When mesoporous catalysts (pore diameter ranging from 2-50 nm, according to IUPAC) are used under conditions close to the boiling point and the associated saturated vapor pressure of a liquid, both liquid and vapor are present in the mesopores, because capillary condensation and evaporation may occur in some pores, depending on their diameter. Because of the different contact angles of condensing liquid and evaporating liquid on a surface ${ }^{1,2}$, the degree of wetting of a catalyst pellet can be different at the same operating conditions, depending on whether the pellet follows the course of continuous condensation or evaporation. As a result of the different diffusion and reaction rates in vapor and liquid filled pores, the apparent catalyst activities, and thus the effectiveness factors, are different. This causes complicated hysteresis phenomena, such as inconsistent steady state branches, observed in experiments ${ }^{3-7}$ when changing reaction conditions in different directions. Understanding these hysteresis phenomena is essential in designing catalysts for multiphase reactions, as well as gas/liquid/solid reactors, such as trickle-bed reactors. ${ }^{8,9}$

A somewhat similar phenomenon of hysteresis due to capillary condensation, which has been studied extensively, is observed in the different nitrogen adsorption and desorption isotherms of porous sorbents and catalyst supports at $77 \mathrm{~K}$, the normal boiling point of nitrogen. Seaton and his coworkers ${ }^{10-13}$ determined pore network connectivity from the adsorption/desorption hysteresis by using percolation theory in which the percolation threshold is tightly linked to the connectivity. Ramírez et al. ${ }^{14}$ and Ramírez and Sierra ${ }^{15}$ found that PSD and pore network connectivity could influence the extent of hysteresis, by running simulations with a numerically constructed pore 
network model. For catalytic reactions accompanied by capillary condensation, it can be more difficult to interpret the effect of pore network structure on hysteresis, because of the compounded complexity of local vapor-liquid equilibrium in individual pores, different reaction rates in liquid phase and vapor phase, and different pathways for diffusion.

To understand the influences of pore network structure on the hysteresis phenomena of multiphase reactions, a practical approach is to experiment with model catalysts that have controllable pore network structures. Hessari and Bhatia ${ }^{6}$ probed the effects of a bimodal pore size distribution, namely a structure containing both mesopores and macropores, on the apparent reaction rate for hydrogenation of cyclohexene in $\mathrm{Pd} / \gamma-\mathrm{Al}_{2} \mathrm{O}_{3}$ catalysts. They observed a sharper evaporation branch of the reaction rate hysteresis loop when the bimodal structure contained more macropores. $\mathrm{Wu}$ et al. ${ }^{16}$ evaluated the effects of pellet radius on the apparent reaction rate for hydrogenation of benzene in $\mathrm{Ni} / \gamma-\mathrm{Al}_{2} \mathrm{O}_{3}$ catalysts. They found that the reaction rate hysteresis loop expands when increasing the pellet radius, and attributed this to the effects of a bimodal pore structure. Nevertheless, a general conclusion cannot be drawn for other pore structures. For this reason, simulations using a pore network model with variable structural parameters is an easier way to obtain more general conclusions, and provides more fundamental information to understand the complex process in a catalyst pellet.

Wood and Gladden ${ }^{17}$ and Wood et al. ${ }^{18}$ used a pore network model to investigate the influences of network topology and pore size distribution on the effectiveness factor for hydrodesulphurization of diethyl sulphide in catalyst pellets. However, they did not consider the different critical radii of condensation and evaporation at the same temperature and pressure, and they did not show how the pore network structure affects hysteresis of the effectiveness factor. In addition, reaction and diffusion in liquid-filled pores and in vapor-filled regions trapped by liquid 
were neglected, whereas, for some catalytic reactions, the reaction rate is considerable, even when a catalyst is totally filled by liquid.

Recently, we have shown that even a simplified pore network model (3D, cubic topology) can predict the experimentally observed hysteresis of the effectiveness factor very well ${ }^{19}$. The key is to consider the different critical radii of condensation and evaporation, as well as the reaction and diffusion in the liquid-filled pores. An additional bonus of this approach is that the distribution of liquid is clearly visualized, explicitly showing the pore blocking phenomenon that contributes significantly to the unstable behavior of catalysts for multiphase reactions. However, our previous work did not investigate the dependence of the hysteresis loop on various parameters, like pore network connectivity, or describe the effects of a bimodal pore size distribution and the spatial distribution of pores (uniform or not), all of which are very important in practice to understand multiphase catalytic processes.

In this article, by using our pore network model, the influence on the hysteresis loop of the different parameters characterizing the pore network structure, such as the pore size distribution, the pore connectivity, the spatial distribution of the pore size, a bimodal pore size distribution, and pellet size are quantified and explained. The results provide valuable insights to help the interpretation of nonlinear hysteresis phenomena for multiphase reactions. It also provides useful guidelines for the optimal design of porous catalysts, as essential input to multiphase reactor design.

\section{Modeling}

\section{Model assumptions}

Hydrogenation of benzene to cyclohexane over $\mathrm{Pd} / \gamma-\mathrm{Al}_{2} \mathrm{O}_{3}$ is chosen as the reaction system due to its industrial and academic significance. Because we concentrate only on the process inside the catalyst pellet, we can neglect any external mass transfer limitations. The temperature gradient in 
the catalyst pellet is assumed negligible, because of the large thermal conductivity ${ }^{20}$ and small size $\left(R_{P} \sim 4 \mathrm{~mm}\right)$ of the pellet ${ }^{19}$. The internal mass transfer rate is assumed to be governed by molecular diffusion and Knudsen diffusion, based on other studies that have been experimentally validated ${ }^{7}$. The pressure gradient in the catalyst is negligible $\mathrm{e}^{4,7}$ even when its radius is larger than $8 \mathrm{~mm}^{21}$, so that viscous flow contributions are excluded from description of transport in macropores, while also transport through mesopores is dominated by diffusion ${ }^{4,7}$. The model reaction system is treated as a binary mixture (cyclohexane and benzene as one component mixed with hydrogen) when describing diffusion and capillary condensation, because of the close physical properties of cyclohexane and benzene. We refer to our previous article in the Journal for more details on the $\operatorname{model}^{19}$.

\section{Pore network}

A three-dimensional pore network with a regular topology is used to represent the pore network structure of a porous catalyst particle (see Fig. 1S). This pore network is confined in a spherical domain to reflect the shape of a real spherical catalyst particle commonly used in industry. The nodes are treated as zero-volume intersections, and are connected by cylindrical pores to their neighbors, according to the connectivity, which is assumed to be at most 6 (the connectivity of the original, fully connected cubic lattice). The radius of each pore is assigned to meet predefined statistical and spatial distributions. The cylindrical pore shape could easily be substituted by a more

realistic morphology, such as a tortuous channel with fractal surface roughness ${ }^{22,23}$, however this requires additional parameters that should be derived from experimental characterization data.

\section{Reaction and diffusion}


The pore model is similar to the one in our previous paper ${ }^{19}$; we therefore only summarize the principal equations.

The continuity equation for component $i$ in a single pore is:

$$
\frac{d J_{i, n}}{d l_{n}} \frac{r_{n}}{2}-R_{i}=0
$$

where $R_{i}$ is the reaction rate per pore surface area of component $i$, and $J_{i, n}, l_{n}$ and $r_{n}$ are the diffusion flux of component $i$, the length coordinate and the radius of pore $n$, respectively. Eqs. (2) and (3) below are used to calculate the reaction rate per pore surface area of component $i$ for vapor-filled pores $\left(R_{i, v}\right)$ and liquid-filled pores $\left(R_{i, l}\right)^{7}$, respectively:

$$
\begin{gathered}
R_{i, v}=\frac{0.199 v_{i} \exp \left(\frac{-28250}{R T}\right) \frac{P_{B}}{10}\left(\frac{P_{H}}{10}\right)^{0.5} \frac{1}{S}}{\left[1+1.80 \times 10^{-4} \exp \left(\frac{41170}{R T}\right) \frac{P_{B}}{10}\right]\left[1+2.95 \times 10^{-2} \exp \left(\frac{-9370}{R T}\right)\left(\frac{P_{H}}{10}\right)^{0.5}\right]} \\
R_{i, l}=0.436 v_{i} \exp \left(\frac{-43880}{R T}\right) \frac{P_{H}}{10} \frac{1}{S}
\end{gathered}
$$

Here, $v_{i}$ is the stoichiometric coefficient of component $i, P_{B}$ is the partial pressure of benzene, $P_{H}$ is the partial pressure of hydrogen, $R$ is the universal gas constant, and $S=246.6 \mathrm{~m}^{2} / \mathrm{g}$ is the surface area of the $\mathrm{Pd} / \gamma-\mathrm{Al}_{2} \mathrm{O}_{3}$ catalyst. $^{7}$ For the same reaction conditions, the rate of reaction in the liquid phase is smaller than in the gas phase, because hydrogen is dissolved. $J_{i, n}$ is calculated by Fick's law, because a pseudo-binary mixture can be assumed for this reaction system ${ }^{24}$ :

$$
J_{i, n}=-D_{i, n} \frac{d C_{i, n}}{d l_{n}}
$$

Here, $D_{i, n}$ and $C_{i, n}$ are the effective diffusivity and the concentration of component $i$ in pore $n$, respectively. $D_{i, n}$ for vapor-filled and liquid-filled pores are calculated by the Bosanquet equation, combined with the Wilke approximation ${ }^{21,25}$ and the Tyn and Calus method ${ }^{26}$, respectively. 
For the inner nodes, Kirchhoff's law is used:

$$
\sum_{n=1}^{n=Z} \pi r_{n}^{2} J_{i, n}=0
$$

where $Z$ is the connectivity. For the boundary nodes, Dirichlet boundary conditions are given:

$$
C_{i, n}=C_{i, b}
$$

where $C_{i, b}$ is the bulk concentration of component $i$.

\section{Phase change}

The critical pore radius of capillary condensation $\left(r_{c}\right)$ is calculated by Eq. (7):

$$
r_{c}=t+r_{k}
$$

where $t$ is the thickness of the adsorption layer that can be calculated by using a modified Halsey equation $^{27}$ and $r_{k}$ is the Kelvin radius, calculated by:

$$
\mathrm{r}_{k}=\frac{b M \delta}{R T \rho \ln \left(\frac{P_{s}}{P}\right)}
$$

where $b$ is 1 for condensation and 2 for evaporation, $M$ is the average molecular weight of the adsorbate, $\delta$ is the surface tension of the adsorbate, and $\rho$ is the density of the adsorbate.

A pore is filled with vapor or liquid, according to whether its radius is larger or smaller than $r_{c}$. However, during evaporation, liquid in a pore may not evaporate if it is prevented to do so by liquid in its adjacent pores, which we call "pore blocking” (see Fig. 2S) ${ }^{28-30}$. An extended HoshenKopelman (H-K) algorithm ${ }^{31}$ is used to determine pore blocking, due to the clustering of liquidfilled pores.

\section{Implementation}


The simulation is implemented in MATLAB 2010b using the algorithm illustrated in Fig. 3S. The simulation starts with calculating mass transfer and reaction in the vapor-filled pore network. Then, capillary condensation and pore blocking are applied to identify the liquid-filled pores. The simulation runs repeatedly with reaction and diffusion in both liquid phase and vapor phase with the updated phase distribution in the pore network, until convergence. Finally, the effectiveness factor $(\eta)$ of the catalyst is calculated:

$$
\eta=\frac{\sum_{n=1}^{N} 2 \pi r_{n} l_{n} R_{C}\left(C_{B, n}, C_{H, n}, T\right)}{\sum_{n=1}^{N} 2 \pi r_{n} l_{n} R_{C}\left(C_{B, b}, C_{H, b}, T\right)}
$$

where $N$ is the total number of pores and $R_{C}$ is the rate of producing cyclohexane per unit pore surface area.

The number of nodes in the pore network simulations is 5649; a higher number did not have appreciable effects on the results (Fig. 4S) ${ }^{19}$. The specific pore volume of all model catalysts is $0.47 \mathrm{~cm}^{3} / \mathrm{g}{ }^{7}$ The particle temperature $(415-460 \mathrm{~K})$ and bulk pressure of benzene at the catalyst surface (2.5-7.5 bar) cover the range of research interest, where hysteresis phenomena occur. The pressure of cyclohexane at the external catalyst surface is 0 bar, and the total pressure is 10 bar.

\section{Results and Discussion}

The area of a hysteresis loop reflects the effects of the pore network structure on the multiphase reaction. It can be calculated by Eq. (10) and (11), for bulk benzene pressure and temperature effects, respectively:

$$
\begin{gathered}
S_{P}=\int\left(\eta_{c, P}-\eta_{e, P}\right) d P_{B, b} \\
S_{T}=\int\left(\eta_{c, T}-\eta_{e, T}\right) d T
\end{gathered}
$$


where $\eta_{c}$ and $\eta_{e}$ are the effectiveness factors of the condensation branches (see the solid lines in Fig. 1) and evaporation branches (see the dashed lines in Fig. 1), respectively.

The pore network structure affects the hysteresis loop by directly changing capillary condensation and pore blocking, which are believed to be the root causes of the hysteresis ${ }^{4,5}$ and cause the differences in surface areas accessible to vapor or internal volumes filled by vapor (see Fig. 5S). To decouple the influences of capillary condensation and pore blocking on the hysteresis, we exclude the extended Hoshen-Kopelman algorithm ${ }^{31}$ from the pore network model to obtain evaporation branches with pore blocking excluded (see dotted lines in Fig. 1). The loop area caused by capillary condensation alone $\left(S_{C_{-} P}\right.$ and $\left.S_{C C_{-} T}\right)$ is the area between the condensation branch (solid line) and evaporation branch with pore blocking excluded (dotted line); the loop area caused by pore blocking $\left(S_{P B_{-} P}\right.$ and $\left.S_{P B_{-} T}\right)$ is the area between the two evaporation branches. The percentage of the loop area caused by pore blocking $\left(f_{P B}=S_{P B_{-} P} / S_{P}\right.$ or $\left.f_{P B}=S_{P B_{-} T} / S_{T}\right)$ reflects the level of importance of pore blocking in affecting the hysteresis.

\section{Influence of global parameters describing the pore network structure}

The influence of the volume-averaged pore radius $\left(r_{a}\right)$, standard deviation $(\sigma)$, connectivity $(Z)$, and pellet radius $\left(R_{P}\right)$ are studied in this section. The radius of each pore is randomly assigned, according to a log-normal distribution for the pore radius $(r)^{7}$ :

$$
f(r)=\frac{V_{t}}{\sqrt{2 \pi} r \sigma} \exp \left[-\frac{\left(\ln r-\ln r_{a}\right)^{2}}{2 \sigma^{2}}\right]
$$

where $V_{t}$ is the specific pore volume of the catalyst particle. To avoid undersized or oversized pores, pore radii are limited within a reasonable range $\left[r_{l o w}, r_{h i g h}\right]$, where the values of the cumulative distribution function of the log-normal distribution are between 0.001 and 0.999 . 
Fig. 2 displays the hysteresis loops of the catalyst pellet with different volume-averaged pore radius. The hysteresis loops are characterized by their area, range, and steepness, which represent usage of catalyst and stability of operation. The hysteresis loop area $\left(S_{P}\right.$ or $\left.S_{T}\right)$ decreases from 0.57 bar to 0.19 bar in Fig. $2 \mathrm{a}$ and from $5.5 \mathrm{~K}$ to $1.3 \mathrm{~K}$ in Fig. $2 \mathrm{~b}$, with the increase of volume-averaged pore radius from $2.5 \mathrm{~nm}$ to $10 \mathrm{~nm}$. The loop area is shrunk, because capillary conditions (pressure and temperature) for evaporation are closer to the ones for condensation when the pore radius is larger, according to Eq. (8). For example, if most pores in the pore network are macropores $(r>25$ $\mathrm{nm})$, the hysteresis loop area approaches 0 , because the evaporation branch of the effectiveness factor is very close to the condensation branch. Besides, as seen from the tables in Fig. 2, the loop area caused by pore blocking $\left(S_{P B_{-} P}\right.$ or $\left.S_{P B_{-} T}\right)$ also decreases proportionally with the loop area caused by capillary condensation alone $\left(S_{C_{-} P}\right.$ or $\left.S_{C C_{-} T}\right)$, which also contributes to the shrinking of the total loop area.

Fig. 2 also shows that the hysteresis loop moves towards a high bulk pressure of benzene (see Fig. 2a) and a low particle temperature (see Fig. 2b) when increasing the volume-averaged pore radius. The hysteresis loop covers the same pressure and temperature ranges where capillary condensation occurs. The larger volume-averaged pore radius requires a higher pressure of benzene and lower temperature for capillary condensation, according to Eq. (8).

It is worth noting that a non-monotonous change of the effectiveness factor with the particle temperature is observed in Fig. $2 \mathrm{~b}$ as well as in Figs. 3b-8b. If one phase (e.g., liquid) is dominant in the pore network, the effectiveness factor decreases with the increase of temperature, because diffusion is less sensitive to temperature than reaction kinetics. But when the temperature is high enough and the liquid in some of the pores is evaporated, the effectiveness factor increases significantly because the reaction rate in the vapor phase is much larger than the one in the liquid 
phase. After most of the liquid is evaporated, increasing further the temperature would again decrease the effectiveness factor.

In Fig. 3, when increasing the standard deviation from 0.1 to 0.5 , the hysteresis loop area increases from 0.32 bar to 0.50 bar in Fig. $3 \mathrm{a}$ and from $2.3 \mathrm{~K}$ to $3.9 \mathrm{~K}$ in Fig. $3 \mathrm{~b}$. As seen from the tables in Fig. 3, a wide pore size distribution strengthens pore blocking effects $\left(f_{P B}\right)$ and also increases the loop area caused by capillary condensation alone $\left(S_{C C_{-} P}\right.$ or $\left.S_{C C_{-} T}\right)$, which is responsible for the enlarged total loop area. Besides, when increasing the standard deviation, the hysteresis loop expands towards the low bulk pressure of benzene and high particle temperature. Higher standard deviation indicates more small pores, for which a lower pressure and higher particle temperature are sufficient to induce capillary condensation.

Fig. 4 shows how the pore connectivity affects the hysteresis loop. When increasing the connectivity from 3 to 5 , the hysteresis loop area decreases from 0.43 bar to 0.36 bar in Fig. $4 \mathrm{a}$ and from $3.1 \mathrm{~K}$ to $2.6 \mathrm{~K}$ in Fig. 4b. The hysteresis loop area is shrunk, because an increased pore connectivity reduces the chances for pore blocking (see $f_{P B}$ in the tables in Fig. 4). However, the loop area cannot be shrunk further, if the pore connectivity is sufficiently high (in this case: $Z=5$ ). The condensation branches for the three connectivities are very close. During condensation, the effectiveness factor is only affected by capillary condensation and diffusion; the connectivity cannot change capillary condensation and only slightly influences diffusion in this reaction system. Similar arguments will be made when discussing the results shown in Figure 6 and 7.

Fig. 5 shows how the pellet radius affects the hysteresis loop. When increasing the pellet radius from $2 \mathrm{~mm}$ to $8 \mathrm{~mm}$, the hysteresis loop area stays around 0.37 bar in Fig. 5a and 2.7 K in Fig. 5b, although the hysteresis loop obviously moves down, because the lengthened diffusion route reduces the effectiveness factor. The hysteresis loop area is insensitive to the pellet radius $\left(R_{p}<10\right.$ 
$\mathrm{mm}$ ) commonly used for industrial porous catalysts. This indicates that the hysteresis loop area is not significantly affected by diffusion alone. However, this loop area can be significantly influenced by capillary condensation and pore blocking, as already shown in Fig. 2-4.

\section{Influences of spatial distributions of pore size}

Three archetypical spatial distributions of pore $\operatorname{size}^{32}$ are considered in this work. These distributions are a random spatial distribution of pore size (SD1), pore size descending towards the center of the spherical catalyst particle (SD2), and pore size ascending towards the center of the spherical catalyst particle (SD3), as shown in the inset of Fig 6. To ensure that the spatial distribution is the only variable, volume averaged pore size $\left(r_{a}=5 \mathrm{~nm}\right)$, standard deviation $(\sigma=0.3)$, connectivity $(Z=4)$, and pellet radius $\left(R_{p}=4 \mathrm{~mm}\right)$ are all kept constant.

As seen in Fig. 6, when large pores are located in the zone close to the catalyst surface (i.e., SD2), the hysteresis loop area is the smallest one (i.e., 0.34 bar in Fig. 6a and $2.5 \mathrm{~K}$ in Fig. 6b), because there is no pore blocking effect $\left(f_{P B}=0\right.$, see the tables in Fig. 6). When the largest pores are located in the central zone of the catalyst (i.e., SD3), the hysteresis loop area is the largest one (i.e., 0.54 bar in Fig. 6 a and $4.5 \mathrm{~K}$ in Fig. 6b), because the pore blocking effect is the strongest (see $f_{P B}$ in the tables in Fig. 6). Therefore, the hysteresis loop area is very sensitive to spatial distributions of pore size.

\section{Influence of bimodal pore size distribution}

A bimodal pore size distribution is characteristic for many real porous catalyst pellets, commonly consisting of mesopores and macropores. A bimodal pore size distribution of the model catalysts is generated by performing the following two steps: first, randomly assigning a pore radius to each pore, according to a log-normal distribution (i.e., $r_{a}=5 \mathrm{~nm}$ and $\sigma=0.3$ ), as described 
by Eq. (12), to build a unimodal mesoporous structure; second, replacing some mesopores with macropores (constant macropore radius: $50 \mathrm{~nm}$ ) to form a bimodal structure. The value of $50 \mathrm{~nm}$ is quite arbitrary and does not qualitatively affect the results, because, in contrast to mesopores, the capillary condensation conditions for macropores with different pore radii are almost the same. The macropores are wide enough not to lead to noticeable viscous flow resistance.

Two spatial distributions of macropores in the bimodal structures are compared. They are a random spatial distribution of macropores (BD1) and a spatial distribution (BD2) where all macropores form a cluster that is directly connected to the outer surface of the catalyst, as shown in the inset of Fig. 7. Apart from this, the effects of the fraction of macropores $\left(f_{M}\right)$ in the bimodal structures are also investigated.

Fig. 7 compares the hysteresis loops corresponding to the two distributions. The hysteresis loop area of $\mathrm{BD} 1$ is much larger than the one of $\mathrm{BD} 2$. This is to be expected, since, for BD1, the pore blocking effect is more significant, because the liquid in some macropores might be blocked by the liquid in adjoining mesopores (see $f_{P B}$ in the tables in Fig. 7); for BD2, the pore blocking effect is less important because all the liquid in the macropores is ultimately connected to the exterior, thus can readily evaporate. When most macropores are evacuated, the influence of a spatial distribution of macropores vanishes, so that the evaporation branches for BD1 and BD2 partially overlap.

Fig.8 shows how the percentage of macropores in a random bimodal structure changes the hysteresis loop. For $f_{M}=5 \%$ and $f_{M}=25 \%$, the area of the hysteresis loop is very close (around 0.43 bar in Fig. 8a and $2.8 \mathrm{~K}$ in Fig. 8b), because of two competing factors. When $f_{M}$ is small, the contribution of capillary condensation (see $S_{C C_{-} P}$ or $S_{C C_{-} T}$ in the tables in Fig. 8) to the hysteresis loop area is relatively important, but the contribution of pore blocking (see $S_{P B_{-} P}$ or $S_{P B_{-} T}$ ) is relatively small; when $f_{M}$ is larger, but not too large, the contribution of capillary condensation is 
relatively small but the contribution of pore blocking is relatively large. If $f_{M}$ is very large (e.g., $f_{M}=50 \%$ ), beyond the percolation threshold ${ }^{33}$, the macropores form percolation clusters, and the loop area caused by pore blocking $\left(S_{P B_{-} P}\right.$ or $\left.S_{P B_{-} T}\right)$ as well as capillary condensation $\left(S_{C C_{-} P}\right.$ or $\left.S_{C C_{-} T}\right)$ decreases significantly, despite of the increase in the proportion of the loop area caused by pore blocking, $f_{P B}$. Therefore, the hysteresis loop area for $f_{M}=50 \%$ is the smallest one, i.e., 0.29 bar in Fig. $8 \mathrm{a}$ and $1.8 \mathrm{~K}$ in Fig. $8 \mathrm{~b}$.

\section{Conclusions}

In this work, a pore network model has been employed to simulate coupled reaction, diffusion, capillary condensation, and pore blocking in $\mathrm{Pd} / \gamma-\mathrm{Al}_{2} \mathrm{O}_{3}$ catalysts for the catalytic hydrogenation of benzene to cyclohexane. The effects of various parameters characterizing the pore network structure on this multiphase reaction were quantified by measuring the hysteresis loop of the effectiveness factor.

When the volume-averaged pore radius is small, the pore size distribution is wide, and the connectivity of the pore network is poor, the hysteresis loop expands to a large extent, because of the increased contribution of capillary condensation and pore blocking. However, a catalyst with a large volume-averaged pore radius and narrow pore size distribution has steep hysteresis loops, which may not be favorable for the operation of gas/liquid/solid reactors. The pellet radius only changes the overall diffusion rates, but, interestingly, does not influence the hysteresis. The spatial distribution of pore size, especially in a pore network with a bimodal pore size distribution, changes the hysteresis loop area significantly, due to influences on capillary condensation and pore blocking.

The spatial distribution of active sites ( $\mathrm{Pd}$ component in this work) over the catalyst pellet may also affect the hysteresis loops through changing the local reaction kinetics ${ }^{34}$, a subject worthy of 
investigation. In addition, due to the change of the volume-based activity with the volumeaveraged pore radius and distribution, when optimizing industrial catalysts for optimal performance in reactors, a compromise among the effective usage of the catalyst (effectiveness factor), the stability of operation (steepness and area of the hysteresis loop), and the reactor volume (the volume-based activity of catalyst) is necessary. For this purpose, the effects discussed in this paper should be integrated in multiphase reactor simulations and optimization.

\section{AUTHOR INFORMATION}

\section{First Corresponding Author}

*Tel.: +86-21-64253509. Fax: +86-21-64253528. E-mail: xgzhou@ecust.edu.cn

\section{Second Corresponding Author}

†Tel.: +44-20-31081126. Fax.: +44-20-73832348. Email address: m.coppens@ucl.ac.uk

\section{Acknowledgments}

The authors are grateful for the financial support of the National Basic Research Program of China (2012CB720501) and the National Natural Science Foundation of China (91434117), as well as support from the UK's EPSRC "Frontier Engineering" Centre for Nature Inspired Engineering (EP/K038656/1). G. Y. is supported by the China Scholarship Council (CSC) for his research at UCL.

\section{Notation}

$C_{i, n} \quad$ concentration of component $i$ in pore $n, \mathrm{~mol} / \mathrm{m}^{3}$

$\mathrm{C}_{i, b}$ bulk concentration of component $i, \mathrm{~mol} / \mathrm{m}^{3}$ 
$D_{i, n} \quad$ effective diffusivity of component $i$ in pore $n, \mathrm{~m}^{2} / \mathrm{s}$

$f(r)$ pore size distribution density at radius $r, \mathrm{~cm}^{3} / \mathrm{g} / \mathrm{nm}$

$J_{i, n}$ diffusion flux of component $i$ in pore $n, \mathrm{~mol} / \mathrm{m}^{2} / \mathrm{s}$

$l_{n} \quad$ length coordinate of pore $n, \mathrm{~m}$

M molecular weight, $\mathrm{g} / \mathrm{mol}$

$P$ pressure, bar

$P_{i} \quad$ partial pressure of component $i$, bar

$P_{i, b} \quad$ bulk pressure of component $i$, bar

$P_{s} \quad$ saturation vapor pressure of the adsorbate, bar

$r \quad$ pore radius, $\mathrm{nm}$

$r_{a} \quad$ volume-averaged pore radius, $\mathrm{nm}$

$r_{c} \quad$ critical pore radius of capillary condensation, $\mathrm{nm}$

$r_{k} \quad$ Kelvin radius, $\mathrm{nm}$

$r_{n} \quad$ radius of pore $n, \mathrm{~nm}$

$R \quad$ universal gas constant, $\mathrm{J} / \mathrm{mol} / \mathrm{K}$

$R_{p} \quad$ catalyst pellet radius, $\mathrm{mm}$

$R_{i} \quad$ reaction rate per unit pore surface area of component $i, \mathrm{~mol} / \mathrm{m}^{2} / \mathrm{s}$

$R_{i, v} \quad$ reaction rate per unit pore surface area of component $i$ for vapor-filled pores, $\mathrm{mol} / \mathrm{m}^{2} / \mathrm{s}$

$R_{i, l} \quad$ reaction rate per unit pore surface area of component $i$ for liquid-filled pores, $\mathrm{mol} / \mathrm{m}^{2} / \mathrm{s}$

$S \quad$ specific surface area of the catalyst, $\mathrm{m}^{2} / \mathrm{g}$

$t \quad$ layer thickness, nm

$T$ temperature, $\mathrm{K}$

$v_{i} \quad$ stoichiometric number of component $i$, dimensionless 
$V_{t} \quad$ specific pore volume of the model catalyst, $\mathrm{cm}^{3} / \mathrm{g}$

$Z$ pore network connectivity, dimensionless

Greek Letters

$\sigma \quad$ standard deviation, dimensionless

$\delta \quad$ surface tension, $\mathrm{N} / \mathrm{m}$

$\rho \quad$ density, $\mathrm{kg} / \mathrm{m}^{3}$

$\eta \quad$ effectiveness factor, dimensionless

\section{Subscripts and Superscripts}

$B$ benzene

C cyclohexane

$H$ hydrogen

$i, j, n \quad$ species type

$P$ pressure

$T$ temperature

\section{References}

1. Cohan LH. Hysteresis and the capillary theory of adsorption of vapors. JACS. 1944;66:98105.

2. Horikawa T, Do DD, Nicholson D. Capillary condensation of adsorbates in porous materials. Adv. Colloid Interface Sci. 2011;169:40-58.

3. Kim DH, Kim YG. An experimental study of multiple steady states in a porous catalyst due to phase transition. J. Chem. Eng. Japan. 1981;14:311-317. 
4. Jaguste DN, Bhatia SK. Partial internal wetting of catalyst particles: hysteresis effects. AIChE J. 1991;37:650-660.

5. Watson PC, Harold MP. Rate enhancement and multiplicity in a partially wetted and filled pellet: experimental study. AIChE J. 1994;40:97-111.

6. Hessari FA, Bhatia SK. Reaction rate hysteresis in a single partially internally wetted catalyst pellet: experiment and modelling. Chem. Eng. Sci. 1996;51:1241-1256.

7. Zhou Z-M, Cheng Z-M, Li Z, Yuan W-K. Determination of effectiveness factor of a partial internal wetting catalyst from adsorption measurement. Chem. Eng. Sci. 2004;59:4305-4311.

8. Cheng Z, Anter AM, Yuan W. Intensification of phase transition on multiphase reactions. AIChE J. 2001;47:1185-1192.

9. Zhou Z, Cheng Z, Yuan W. Simulating a multiphase reactor with continuous phase transition. Chem. Eng. Sci. 2005;60:3207-3215.

10. Seaton NA. Determination of the connectivity of porous solids from nitrogen sorption measurements. Chem. Eng. Sci. 1991;46:1895-1909.

11. Liu H, Zhang L, Seaton NA. Determination of the connectivity of porous solids from nitrogen sorption measurements - II. Generalisation. Chem. Eng. Sci. 1992;47;4393-4404.

12. Murray KL, Seaton NA, Day MA. Analysis of the spatial variation of the pore network coordination number of porous solids using nitrogen sorption measurements. Langmuir $1998,14: 4953-4954$.

13. Murray KL, Seaton NA, Day MA. An adsorption-based method for the characterization of pore networks containing both mesopores and macropores. Langmuir 1999;15;6728-6737.

14. Ramírez A, Sierra L, Mesa M, Restrepo, J. Simulation of nitrogen adsorption-desorption isotherms. Hysteresis as an effect of pore connectivity. Chem. Eng. Sci. 2005;60:4702-4708. 
15. Ramírez A, Sierra L. Simulation of nitrogen sorption processes in materials with cylindrical mesopores: Hysteresis as a thermodynamic and connectivity phenomenon. Chem. Eng. Sci. 2006;61:4233-4241.

16. Wu S, Cheng Z, Liu P, Yu Y, Jin J, Zhou Z. Pore network effects under the reduction of catalyst size at elevated temperatures. Chem. Eng. Sci. 2013;93:350-361.

17. Wood J, Gladden LF. Modeling diffusion and reaction accompanied by capillary condensation using three-dimensional pore networks. Part 1. Fickian diffusion and pseudo-first-order reaction kinetics. Chem. Eng. Sci. 2002;57:3033-3045.

18. Wood J, Gladden LF, Keil FJ. Modeling diffusion and reaction accompanied by capillary condensation using three-dimensional pore networks. Part 2. Dusty gas model and general reaction kinetics. Chem. Eng. Sci. 2002;57:3047-3059.

19. Ye G, Zhou X, Coppens M.-O. Yuan W. Probing pore blocking effects on multiphase reactions within porous catalyst particles using a discrete model. AIChE J. 2016;62:451-460.

20. Kehoe JPG, Butt JB. Interactions of inter- and intraphase gradients in a diffusion limited catalytic reaction. AIChE J. 1972;18:347-355.

21. Solsvik S, Jakobsen HA. Modeling of multicomponent mass diffusion in porous spherical pellets: application to steam methane reforming and methanol synthesis. Chem. Eng. Sci. 2011;66:1986-2000.

22. Coppens M.-O. The effect of fractal surface roughness on diffusion and reaction in porous catalysts — From fundamentals to industrial applications. Catal. Today 1999;53;225-243.

23. Coppens M.-O. Characterization of fractal surface roughness and its influence on diffusion and reaction. Colloid Surf. A-Physicochem. Eng. Asp. 2001;s187-188;257-265.

24. Krishna R, Wesselingh JA. The Maxwell-Stefan approach to mass transfer. Chem. Eng. Sci. 1997;52:861-911. 
25. Wilke CR. Diffusional properties of multicomponent gases. Chem. Eng. Prog. 1950;46:95104.

26. Poling BE, Prausnitz JM, O’ Connell JP. The properties of gases and liquids (5th edition). Boston: McGraw-Hill, 2001.

27. Androutsopoulos GP, Salmas CE. A new model for capillary condensation-evaporation hysteresis based on a random corrugated pore structure concept: prediction of intrinsic pore size distribution. 2. model application. Ind. Eng. Chem. Res. 2000;39:3764-3777.

28. Mason G. Determination of the pore-size distribution and pore-space interconnectivity of vycor porous glass from adsorption-desorption hysteresis capillary condensation isotherms. Proc. R. Soc. Lond. A. 1988;415:453-486.

29. Rajniak P, Yang RT. A simple model and experiments for adsorption-desorption hysteresis: water vapor on silica gel. AIChE J. 1993;39:774-786.

30. Rajniak P, Yang RT. Hysteresis-dependent adsorption-desorption cycles: generalization for isothermal conditions. AIChE J. 1994;40:913-924.

31. Al-Futaisi A, Patzek TW. Extension of Hoshen-Kopelman algorithm to non-lattice environments. Physica A. 2003;321:665-678.

32. Ye G, Duan X, Zhu K, Zhou X, Coppens M.-O. Optimizing spatial pore-size and porosity distributions of adsorbents for enhanced adsorption and desorption performance. Chem. Eng. Sci. 2015;132:108-117.

33. Stauffer D. Introduction to percolation theory. London: Taylor and Francis, 1985.

34. Morbidelli M, Gavriilidis A, Varma A. Catalyst design: Optimal distribution of catalyst in pellets, reactors, and membranes. Cambridge: Cambridge University Press, 2001. 


\section{List of Figure Captions}

Figure 1. Illustrations of the hysteresis loop areas caused by capillary condensation alone $\left(S_{C C_{-} P}\right.$ or $\left.S_{C C_{-} T}\right)$ and pore blocking $\left(S_{P B_{-} P}\right.$ or $\left.S_{P B_{-} T}\right)$. Simulation parameters: (a) $T=433 \mathrm{~K}, Z=3, R_{p}=4 \mathrm{~mm}$, $r_{a}=5 \mathrm{~nm}$, and $\sigma=0.3$; (b) $P_{B, b}=5 \mathrm{bar}, Z=3, R_{p}=4 \mathrm{~mm}, r_{a}=5 \mathrm{~nm}$, and $\sigma=0.3$; the pore radii are randomly distributed in the pore network. The arrows indicate the direction of a change in bulk pressure of benzene or a change in particle temperature.

Figure 2. Effects of the volume-averaged pore radius on the hysteresis loop. Simulation parameters: (a) $T=433 \mathrm{~K}, Z=4, R_{p}=4 \mathrm{~mm}$, and $\sigma=0.3$; (b) $P_{B, b}=5$ bar, $Z=4, R_{p}=4 \mathrm{~mm}$, and $\sigma=0.3$; the pore radii are randomly distributed in the pore network.

Figure 3. Effects of the standard deviation on the hysteresis loop. Simulation parameters: (a) $T=433 \mathrm{~K}, Z=4, R_{p}=4 \mathrm{~mm}$, and $r_{a}=5 \mathrm{~nm}$; (b) $P_{B, b}=5 \mathrm{bar}, Z=4, R_{p}=4 \mathrm{~mm}$, and $r_{a}=5 \mathrm{~nm}$; the pore radii are randomly distributed in the pore network.

Figure 4. Effects of the connectivity on the hysteresis loop. Simulation parameters: (a) $T=433 \mathrm{~K}$, $R_{p}=4 \mathrm{~mm}, r_{a}=5 \mathrm{~nm}$, and $\sigma=0.3$; (b) $P_{B, b}=5 \mathrm{bar}, R_{p}=4 \mathrm{~mm}, r_{a}=5 \mathrm{~nm}$, and $\sigma=0.3$; the pore radii are randomly distributed in the pore network.

Figure 5. Effects of the pellet radius on the hysteresis loop. Simulation parameters: (a) $T=433 \mathrm{~K}$, $Z=4, r_{a}=5 \mathrm{~nm}$, and $\sigma=0.3$; (b) $P_{B, b}=5 \mathrm{bar}, Z=4, r_{a}=5 \mathrm{~nm}$, and $\sigma=0.3$; the pore radii are randomly distributed across the pore network. 
Figure 6. Effects of spatial distributions of pore size on the hysteresis loop. Simulation parameters: (a) $T=433 \mathrm{~K}, R_{p}=4 \mathrm{~mm}, Z=4, r_{a}=5 \mathrm{~nm}$, and $\sigma=0.3$; (b) $P_{B, b}=5$ bar, $R_{p}=4 \mathrm{~mm}, Z=4, r_{a}=5 \mathrm{~nm}$, and $\sigma=0.3$. The three spatial distributions (i.e., SD1, SD2, and SD3) are shown in the inset.

Figure 7. Effects of spatial distributions of macropores in bimodal pore structures on the hysteresis loop. Simulation parameters: (a) $T=433 \mathrm{~K}, R_{p}=4 \mathrm{~mm}, Z=4, r_{a}=5 \mathrm{~nm}, \sigma=0.3$, and $f_{M}=5 \%$; (b) $P_{B, b}=5$ bar, $R_{p}=4 \mathrm{~mm}, Z=4, r_{a}=5 \mathrm{~nm}, \sigma=0.3$, and $f_{M}=5 \%$. The illustrations of two spatial distributions (i.e., BD1 and BD2) are shown in the inset.

Figure 8. Effects of the percentage of macropores in bimodal pore structures on the hysteresis loop. Simulation parameters: (a) $T=433 \mathrm{~K}, R_{p}=4 \mathrm{~mm}, Z=4, r_{a}=5 \mathrm{~nm}, \sigma=0.3$, and BD1; (b) $P_{B, b}=5$ bar, $R_{p}=4 \mathrm{~mm}, Z=4, r_{a}=5 \mathrm{~nm}, \sigma=0.3$, and $\mathrm{BD} 1$. 
(a)

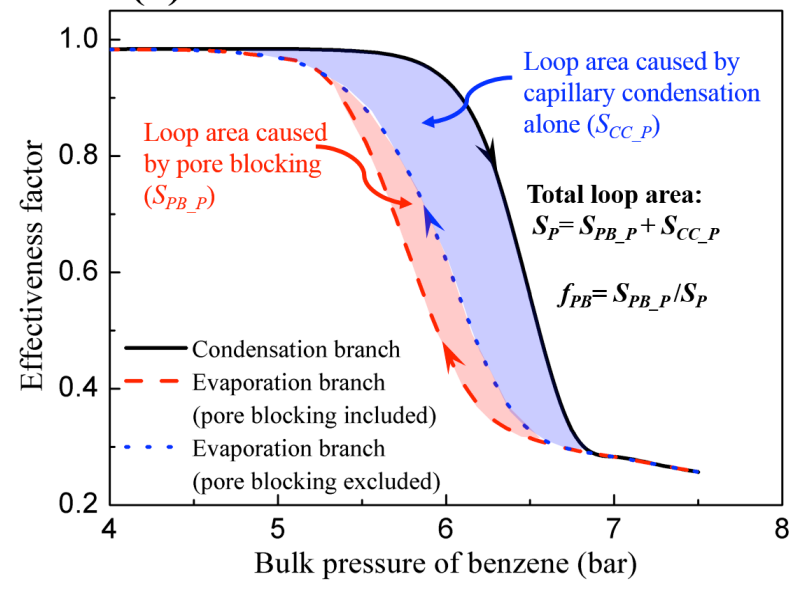

(b)

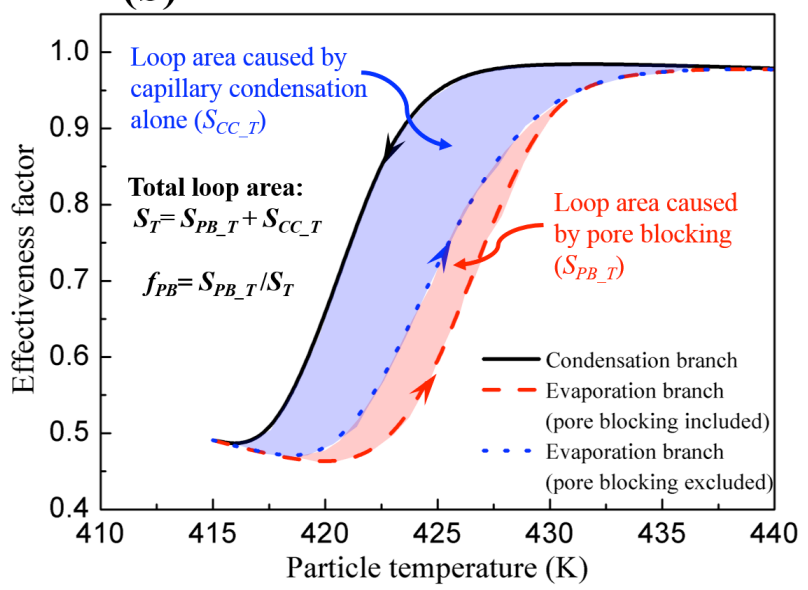

Figure 1. Illustrations of the hysteresis loop areas caused by capillary condensation alone $\left(S_{C C_{-} P}\right.$ or $\left.S_{C C_{-} T}\right)$ and pore blocking $\left(S_{P B_{-} P}\right.$ or $\left.S_{P B_{-} T}\right)$. Simulation parameters: (a) $T=433 \mathrm{~K}, Z=3, R_{p}=4 \mathrm{~mm}$, $r_{a}=5 \mathrm{~nm}$, and $\sigma=0.3$; (b) $P_{B, b}=5 \mathrm{bar}, Z=3, R_{p}=4 \mathrm{~mm}, r_{a}=5 \mathrm{~nm}$, and $\sigma=0.3$; the pore radii are randomly distributed in the pore network. The arrows indicate the direction of a change in bulk pressure of benzene or a change in particle temperature. 

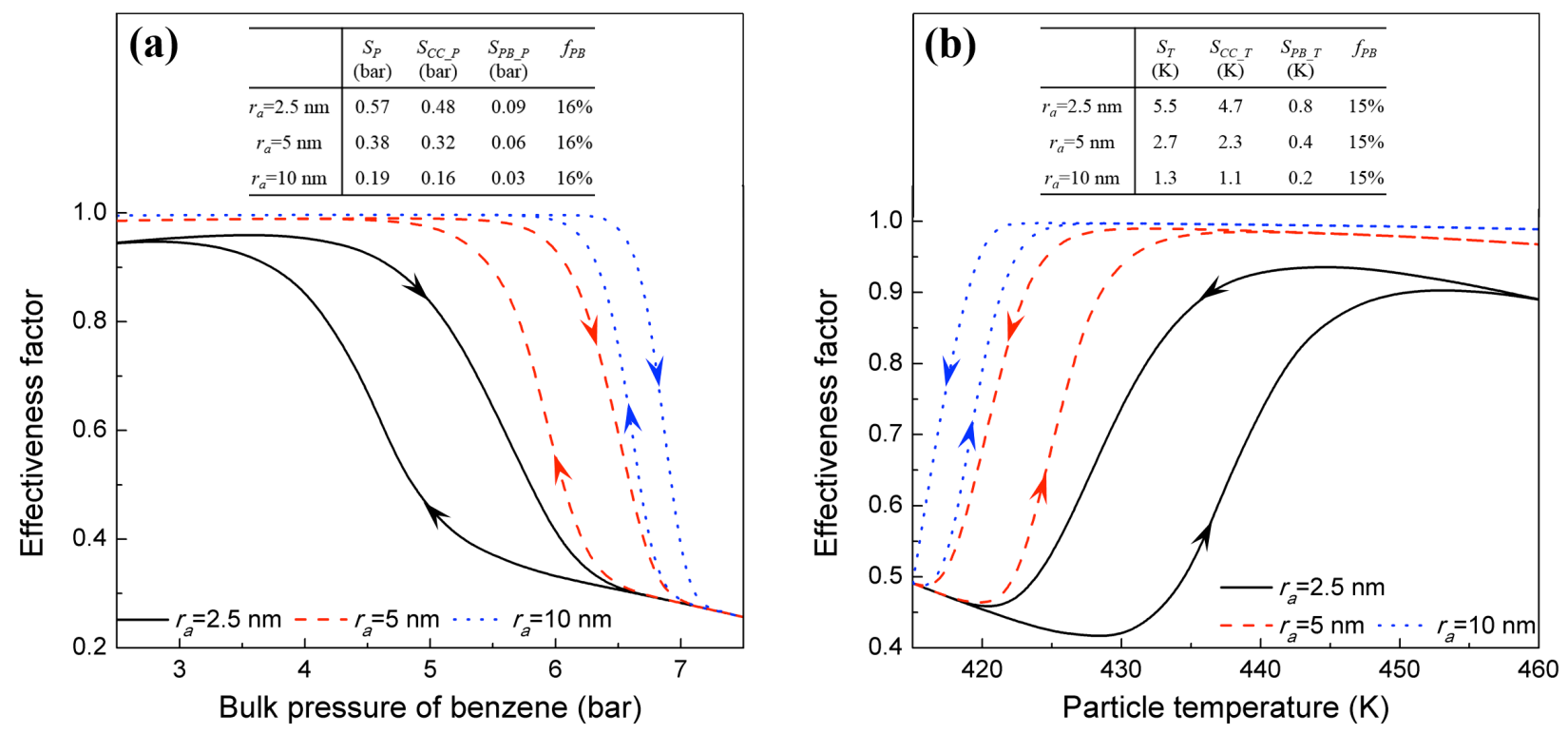

Figure 2. Effects of the volume-averaged pore radius on the hysteresis loop. Simulation parameters: (a) $T=433 \mathrm{~K}, Z=4, R_{p}=4 \mathrm{~mm}$, and $\sigma=0.3$; (b) $P_{B, b}=5$ bar, $Z=4, R_{p}=4 \mathrm{~mm}$, and $\sigma=0.3$; the pore radii are randomly distributed in the pore network. 
(a)

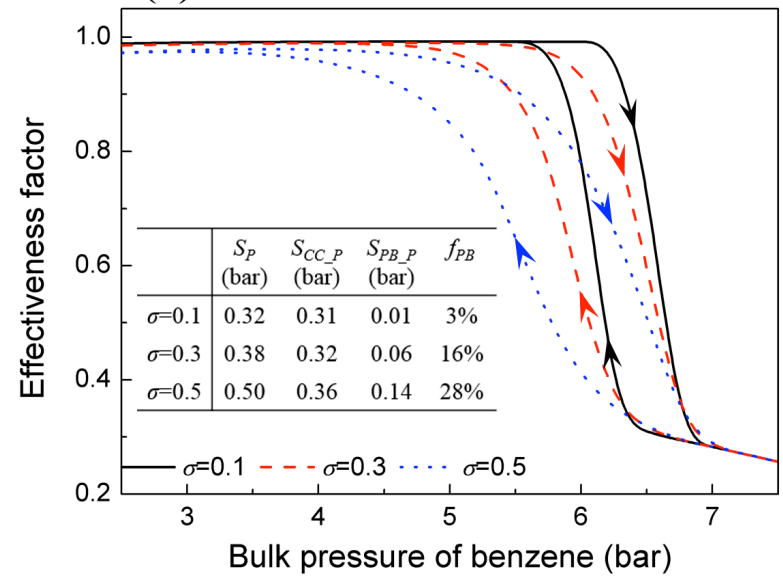

(b)

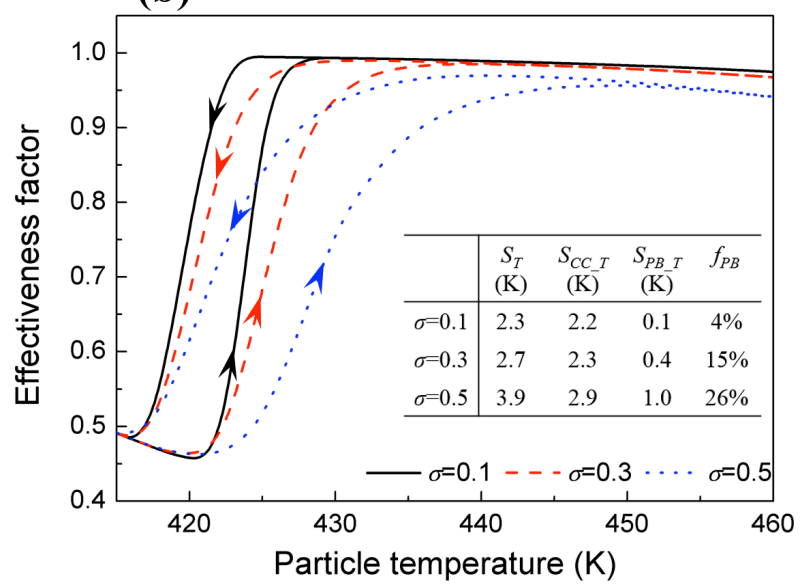

Figure 3. Effects of the standard deviation on the hysteresis loop. Simulation parameters: (a) $T=433 \mathrm{~K}, Z=4, R_{p}=4 \mathrm{~mm}$, and $r_{a}=5 \mathrm{~nm}$; (b) $P_{B, b}=5$ bar, $Z=4, R_{p}=4 \mathrm{~mm}$, and $r_{a}=5 \mathrm{~nm}$; the pore radii are randomly distributed in the pore network. 
(a)

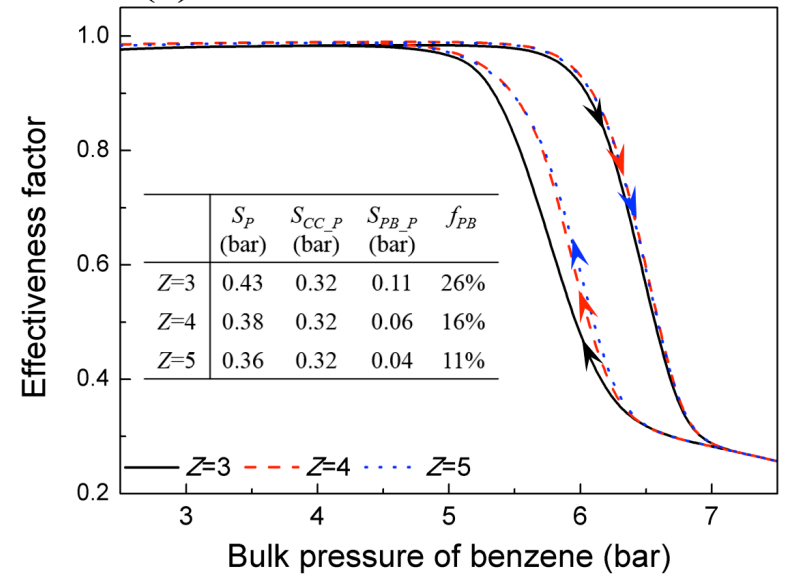

(b)

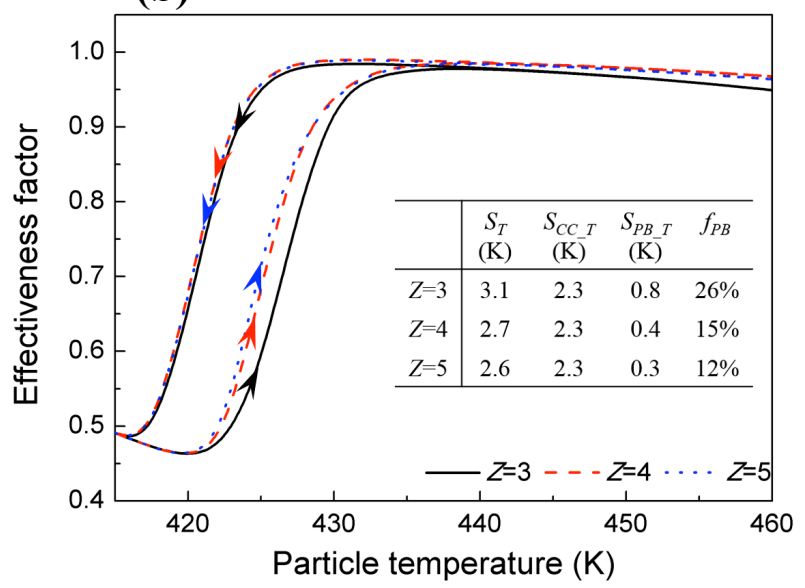

Figure 4. Effects of the connectivity on the hysteresis loop. Simulation parameters: (a) $T=433 \mathrm{~K}$, $R_{p}=4 \mathrm{~mm}, r_{a}=5 \mathrm{~nm}$, and $\sigma=0.3$; (b) $P_{B, b}=5$ bar, $R_{p}=4 \mathrm{~mm}, r_{a}=5 \mathrm{~nm}$, and $\sigma=0.3$; the pore radii are randomly distributed in the pore network. 
(a)

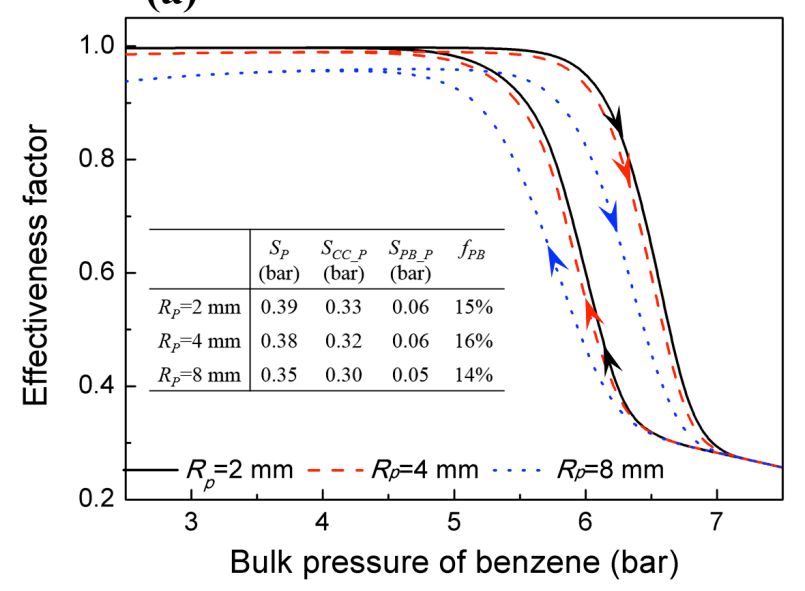

(b)

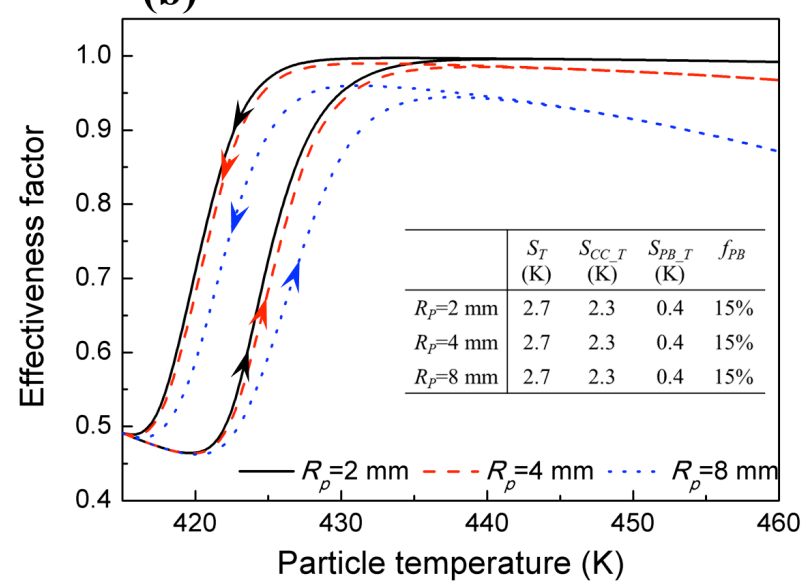

Figure 5. Effects of the pellet radius on the hysteresis loop. Simulation parameters: (a) $T=433 \mathrm{~K}$, $Z=4, r_{a}=5 \mathrm{~nm}$, and $\sigma=0.3$; (b) $P_{B, b}=5 \mathrm{bar}, Z=4, r_{a}=5 \mathrm{~nm}$, and $\sigma=0.3$; the pore radii are randomly distributed across the pore network. 
(a)

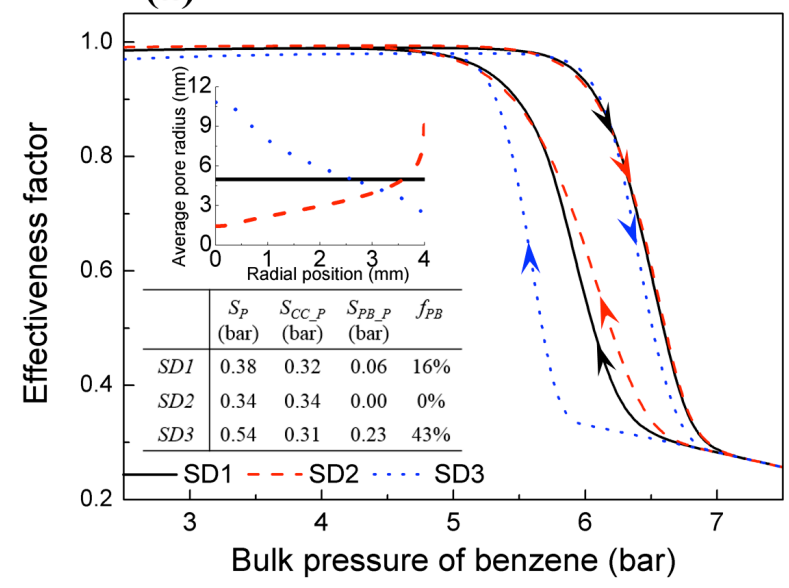

(b)

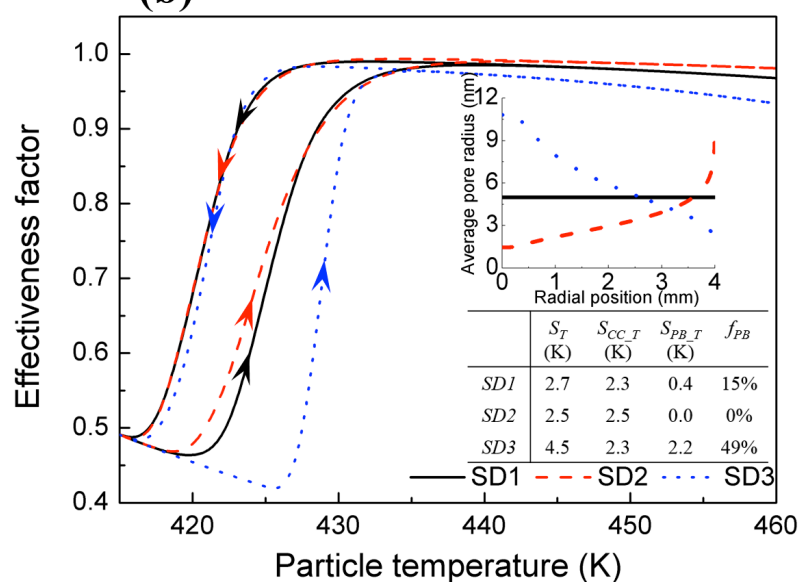

Figure 6. Effects of spatial distributions of pore size on the hysteresis loop. Simulation parameters:

(a) $T=433 \mathrm{~K}, R_{p}=4 \mathrm{~mm}, Z=4, r_{a}=5 \mathrm{~nm}$, and $\sigma=0.3$; (b) $P_{B, b}=5$ bar, $R_{p}=4 \mathrm{~mm}, Z=4, r_{a}=5 \mathrm{~nm}$, and $\sigma=0.3$. The three spatial distributions (i.e., $\mathrm{SD} 1, \mathrm{SD} 2$, and $\mathrm{SD} 3$ ) are shown in the inset. 
(a)

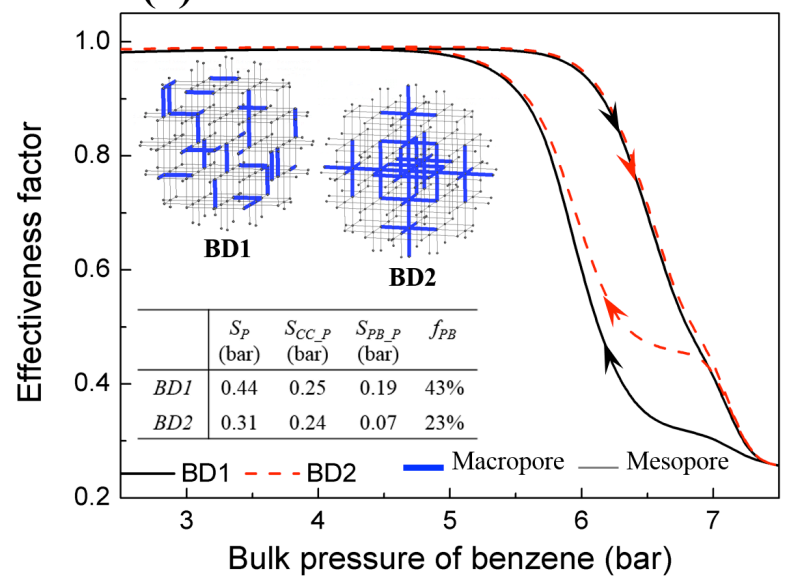

(b)

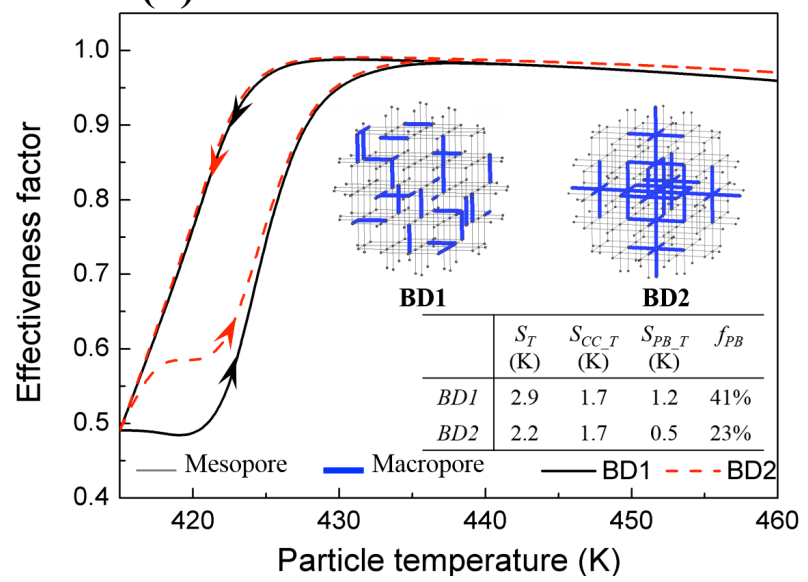

Figure 7. Effects of spatial distributions of macropores in bimodal pore structures on the hysteresis loop. Simulation parameters: (a) $T=433 \mathrm{~K}, R_{p}=4 \mathrm{~mm}, Z=4, r_{a}=5 \mathrm{~nm}, \sigma=0.3$, and $f_{M}=5 \%$; (b) $P_{B, b}=5$ bar, $R_{p}=4 \mathrm{~mm}, Z=4, r_{a}=5 \mathrm{~nm}, \sigma=0.3$, and $f_{M}=5 \%$. The illustrations of two spatial distributions (i.e., $\mathrm{BD} 1$ and $\mathrm{BD} 2)$ are shown in the inset. 
(a)

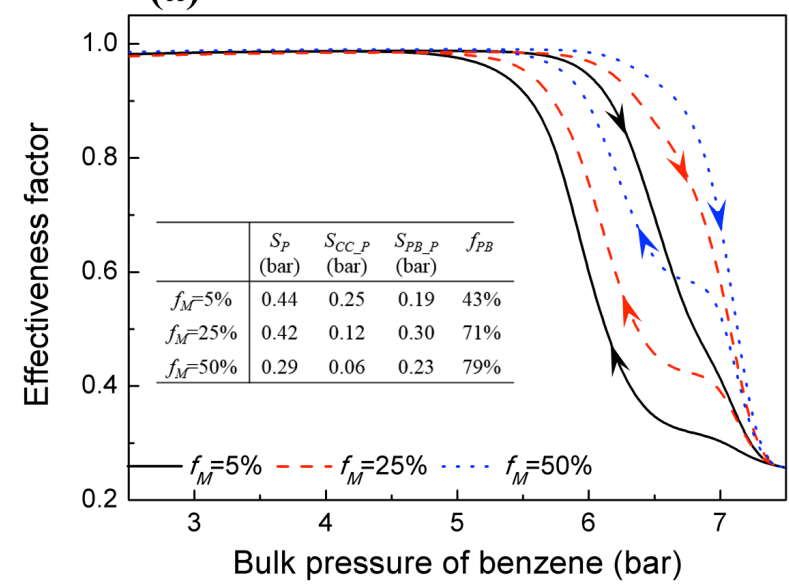

(b)

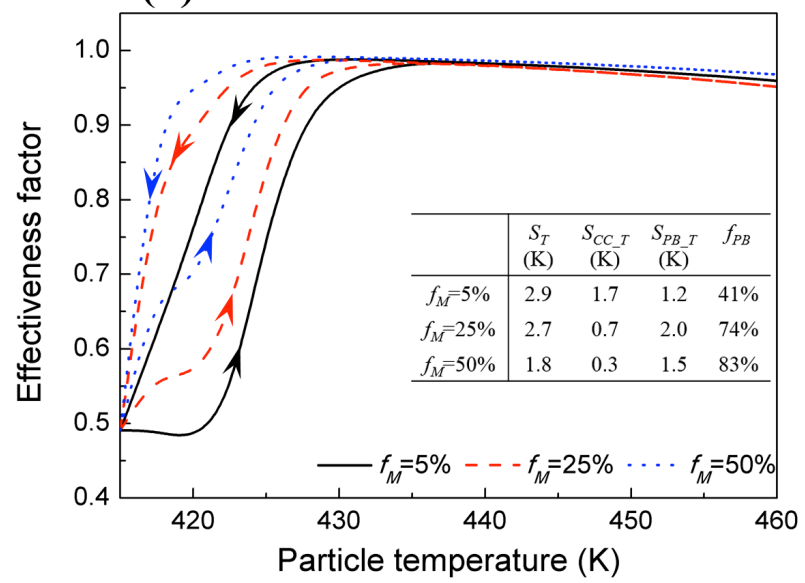

Figure 8. Effects of the percentage of macropores in bimodal pore structures on the hysteresis loop. Simulation parameters: (a) $T=433 \mathrm{~K}, R_{p}=4 \mathrm{~mm}, Z=4, r_{a}=5 \mathrm{~nm}, \sigma=0.3$, and BD1; (b) $P_{B, b}=5$ bar, $R_{p}=4 \mathrm{~mm}, Z=4, r_{a}=5 \mathrm{~nm}, \sigma=0.3$, and BD1. 